\title{
ON THE NONTRIVIAL SOLVABILITY OF SYSTEMS OF HOMOGENEOUS LINEAR EQUATIONS OVER $\mathbb{Z}$ IN ZFC
}

\author{
JAN ŠAROCH
}

\begin{abstract}
Following the paper [7, we investigate in ZFC the following compactness question: for which unountable cardinals $\kappa$, an arbitrary nonempty system $S$ of homogeneous $\mathbb{Z}$-linear equations is nontrivially solvable in $\mathbb{Z}$ provided that each its nonempty subsystem of cardinality $<\kappa$ is nontrivially solvable in $\mathbb{Z}$ ?
\end{abstract}

\section{INTRODUCTION AND PRELIMINARIES}

In what follows, group means always an abelian group, i.e. a $\mathbb{Z}$-module. We say that a system $S$ of homogeneous $\mathbb{Z}$-linear equations with a set $X=\left\{x_{i} \mid i \in I\right\}$ of variables is nontrivially solvable in a group $H$ if there exists a mapping $f: X \rightarrow$ $H \backslash\{0\}$ such that, whenever $\sum_{j \in J} a_{j} x_{j}=0$ is an equation from $S$ (where $J$ is a finite subset of $I$ and $a_{j} \in \mathbb{Z}$ for each $\left.j \in J\right)$, then $\sum_{j \in J} a_{j} f\left(x_{j}\right)=0$ holds in $H$.

This notion of nontriviality is a little bit unusual. If we assume instead that the mapping $f$ goes to $H$ and it is not constantly zero on all $x \in X$ that appear in the system $S$, we say that the system $S$ is weakly nontrivially solvable in $H$. More natural as it might me, this weaker notion has got one significant disadvantage: unlike with nontrivial solvability, if a system $S$ is weakly nontrivially solvable and $T$ is a nonempty subsystem of $S$, then $T$ need not be weakly nontrivially solvable. Notice also that an empty system $S$ is (weakly) nontrivially solvable by definition.

Motivated by the work in [7, our aim is to characterize the class $\mathcal{S}(\mathcal{W S}$, resp.) of all infinite cardinals $\kappa$ such that any system $S$ of homogeneous $\mathbb{Z}$-linear equations is nontrivially (weakly nontrivially, resp.) solvable in $\mathbb{Z}$ provided that each subsystem $T \subseteq S$ of cardinality $<\kappa$ is nontrivially (weakly nontrivially, resp.) solvable in $\mathbb{Z}$. In [7, Section 2.2], the authors present several well-known examples of countable $S$ which show in ZF that $\aleph_{0} \notin \mathcal{S} \cup \mathcal{W S}$. They also discuss various interesting related questions in ZF: among other things, they provide a model of ZF without choice where $\aleph_{1} \notin \mathcal{S}$ while they note that the result is not known in ZFC.

In this short note, we use $\kappa$-free groups with trivial dual to show that ZFC actually proves $\aleph_{n} \notin \mathcal{S}$ for each $n \in \omega$. Moreover, it is consistent with ZFC that $\mathcal{S}=\mathcal{W S}=\varnothing$. On the other hand, we are able to prove that $\kappa \in \mathcal{W S} \cap \mathcal{S}$ whenever there exits a regular $\mathcal{L}_{\omega_{1} \omega}$-compact cardinal below $\kappa$ (see Corollary 2.2 and Theorem 3.2). A detailed discussion is contained in Section 4 .

For an unexplained terminology, we recommend, for instance, the very wellwritten extensive book $\underline{4}$.

Date: December 18, 2018.

2010 Mathematics Subject Classification. 08A45, 13C10 (primary) 20K30, 03E35, $03 \mathrm{E} 55$ (secondary).

Key words and phrases. homogeneous $\mathbb{Z}$-linear equation, $\kappa$-free group, $\mathcal{L}_{\omega_{1} \omega}$-compact cardinal.

This work has been supported by Charles Univ. Research Centre program No. UNCE/SCI/022 and by grant GAČR 17-23112S. 


\section{The CASE OF $\mathcal{S}$}

Recall that, given an uncountable cardinal $\nu$, we say that a cardinal $\kappa$ is $\mathcal{L}_{\nu \omega}$ compact if every $\kappa$-complete filter on any set $I$ can be extended to a $\nu$-complete ultrafilter. Observe that a cardinal $\mu$ is $\mathcal{L}_{\nu \omega}$-compact whenever there exists an $\mathcal{L}_{\nu \omega}$-compact cardinal $\lambda$ such that $\lambda \leq \mu$. This is obviously a large cardinal notion for the existence of an $\mathcal{L}_{\nu \omega}$-compact cardinal implies the existence of a measurable cardinal.

The following result is a classic one. We give a proof for the reader's convenience.

Proposition 2.1. Let $\lambda$ be a regular $\mathcal{L}_{\nu \omega}$-compact cardinal and $\mathcal{Z}$ a structure in a first order language $L$ with $|Z|<\nu$. Then a system $S$ consisting of first order formulas in variables from a set $X$ is realized in $\mathcal{Z}$ provided that each its subsystem $T$ of cardinality $<\lambda$ is realized in $\mathcal{Z}$.

Proof. First, let $E$ denote the set $Z^{X}$ of all mappings from $X$ to $Z$. By the assumption, for each $T \in[S]^{<\lambda}$, there exists $e \in E$ such that $\mathcal{Z} \models \varphi[e]$ for each $\varphi \in T$. Let $\mathcal{F}$ be the filter on $E$ generated by the sets $E_{T}=\{e \in E \mid \mathcal{Z} \models \varphi[e]$ for all $\varphi \in T\}$. Since $\lambda$ is regular, we see that $\mathcal{F}$ is a $\lambda$-complete filter. Let $\mathcal{G}$ denote an extension of $\mathcal{F}$ to a $\nu$-complete ultrafilter.

For each $(x, z) \in X \times Z$, put $E_{x, z}=\{e \in E \mid e(x)=z\}$ and define $f \in Z^{X}$ by the assignment $f(x)=z \Leftrightarrow E_{x, z} \in \mathcal{G}$. We can do it like that since the ultrafilter $\mathcal{G}$ picks, for each fixed $x \in X$, exactly one element from the disjoint partition $E=\bigcup_{z \in Z} E_{x, z} ;$ recall that $|Z|<\nu$.

Now let $\varphi \in S$ be arbitrary and $x_{1}, \ldots, x_{n}$ be variables freely occuring in $\varphi$. Then $\varnothing \neq E_{\{\varphi\}} \cap \bigcap_{i=1}^{n} E_{x_{i}, f\left(x_{i}\right)} \in \mathcal{G}$, and so $f \in E_{\{\varphi\}}$. We conclude that $S$ is realized in $\mathcal{Z}$ using the evaluation $f$.

Corollary 2.2. Let $\kappa$ be a cardinal and $\lambda \leq \kappa$ a regular $\mathcal{L}_{\omega_{1} \omega}$-cardinal. Then every nonempty system $S$ of homogeneous $\mathbb{Z}$-linear equations in variables from a set $X$ is nontrivially solvable in $\mathbb{Z}$ whenever each its nonempty subsystem of cardinality $<\kappa$ is nontrivially solvable in $\mathbb{Z}$. In other words $\kappa \in \mathcal{S}$.

Proof. In the system $S$ replace each equation $\psi$ in variables $x_{1}, \ldots, x_{n} \in X$ by the formula $\psi \& \bigwedge_{i=1}^{n} x_{i} \neq 0$ and use Proposition 2.1.

Before we turn our attention to the negative part, we need one preparatory lemma which holds in the general context of $R$-modules over a (commutative) noetherian domain. Recall, that, for a module $M \in \operatorname{Mod}-R$ and an ordinal number $\sigma$, an increasing chain $\mathcal{M}=\left(M_{\alpha} \mid \alpha \leq \sigma\right)$ of submodules of $M$ is called a filtration of $M$ if $M_{0}=0, M_{\beta}=\bigcup_{\alpha<\beta} M_{\alpha}$ whenever $\beta \leq \sigma$ is a limit ordinal, and $M_{\sigma}=M$.

Lemma 2.3. Let $R$ be an infinite noetherian domain, $M$ a free $R$-module of rank $\mu \geq \aleph_{0}$, and $\mathcal{M}=\left(M_{\alpha} \mid \alpha \leq \sigma\right)$ be a filtration of $M$ where, for all $\alpha<\sigma$, $M_{\alpha+1}=M_{\alpha}+\left\langle a_{\alpha}\right\rangle$ with $a_{\alpha} \in M \backslash M_{\alpha}$. For each $\alpha<\sigma$, let $z_{\alpha} \in R$ be arbitrary.

Then there is a homomorphism $\psi: M \rightarrow R$ such that $\psi\left(a_{\alpha}\right) \neq z_{\alpha}$ for all $\alpha<\sigma$.

Proof. First, assume that $\mu=\aleph_{0}$. Let $\left\{g_{n} \mid n<\omega\right\}$ be a set of free generators of $M$. For each $\alpha<\sigma$, we express $a_{\alpha}$ as $\sum_{n \in I_{\alpha}} b_{n \alpha} g_{n}$, where $I_{\alpha}$ is a finite subset of $\omega$ and $b_{n \alpha} \in R \backslash\{0\}$ for every $n \in I_{\alpha}$.

Using the fact that a free $R$-module of finite rank is noetherian, we infer that, for each $n<\omega$, the set $A_{n}=\left\{\alpha<\sigma \mid I_{\alpha} \subseteq\{0,1, \ldots, n\}\right\}$ is finite. Note that $\sigma=$ $\bigcup_{n<\omega} A_{n}$. On the free generators of $M$, we recursively construct a homomorphism $\psi: M \rightarrow R$ as follows:

Let $\psi\left(g_{0}\right)$ be arbitrary such that, for each $\alpha \in A_{0}, b_{0 \alpha} \psi\left(g_{0}\right) \neq z_{\alpha}$. There is always an applicable choice by the hypothesis on $R$. Assume that $n>0, \psi\left(g_{n-1}\right)$ is defined, and $\psi\left(a_{\alpha}\right) \neq z_{\alpha}$ for each $\alpha \in A_{n-1}$. 
We define $\psi\left(g_{n}\right)$ arbitrarily in such a way that, for each $\alpha \in A_{n} \backslash A_{n-1}$, we have

$$
b_{n \alpha} \psi\left(g_{n}\right) \neq z_{\alpha}-\sum_{k \in I_{\alpha} \backslash\{n\}} b_{k \alpha} \psi\left(g_{k}\right) .
$$

This is possible, since $A_{n} \backslash A_{n-1}$ is finite, $b_{n \alpha} \neq 0$ for each $\alpha$ from this set, and $R$ is an infinite domain. It immediately follows that $\psi\left(a_{\alpha}\right) \neq z_{\alpha}$ for each $\alpha \in A_{n}$.

Now, let $\mu$ be an uncountable cardinal. Again, let $\left\{g_{\beta} \mid \beta<\mu\right\}$ be a set of free generators of $M$, and put $G_{B}=\left\langle g_{\beta} \mid \beta \in B\right\rangle$ for all $B \subseteq \mu$.

We use ideas from [6, Section 7.1]. First, we set $A_{\alpha}=\left\langle a_{\alpha}\right\rangle \leq M$. We say that a subset $S$ of the ordinal $\sigma$ is 'closed' if every $\alpha \in S$ satisfies

$$
M_{\alpha} \cap A_{\alpha} \subseteq \sum_{\beta \in S, \beta<\alpha} A_{\beta} .
$$

Notice that any ordinal $\alpha \leq \sigma$ is a 'closed' subset of $\sigma$. For a 'closed' subset $S$, we define $M(S)=\sum_{\alpha \in S} A_{\alpha}$. The results from [6, Section 7.1] give us the following:

(1) For a system $\left(S_{i} \mid i \in I\right)$ of 'closed' subsets, $\bigcap_{i \in I} S_{i}$ and $\bigcup_{i \in I} S_{i}$ is 'closed' as well.

(2) For $S, S^{\prime}$ 'closed' subsets of $\sigma$, we have $S \subseteq S^{\prime} \Longleftrightarrow M(S) \subseteq M\left(S^{\prime}\right)$.

(3) Let $S$ be a 'closed' subset of $\sigma$ and $X$ be a countable subset of $M$. Then there is a 'closed' subset $S^{\prime}$ such that $M(S) \cup X \subseteq M\left(S^{\prime}\right)$ and $\left|S^{\prime} \backslash S\right|<\aleph_{1}$.

Using the properties listed above, we are going to construct a filtration $\mathcal{N}=$ $\left(M\left(S_{\alpha}\right) \mid \alpha \leq \mu\right)$ of $M$ such that, for each $\alpha<\mu$, a) $S_{\alpha}$ is 'closed', b) $S_{\alpha+1} \backslash S_{\alpha}$ is countable, and c) there exists $B_{\alpha} \subseteq \mu$ such that $G_{B_{\alpha}}=M\left(S_{\alpha}\right)$ and $\alpha \subseteq B_{\alpha}$.

We proceed by the transfinite recursion, starting with $S_{0}=B_{0}=\varnothing$. Let $S_{\alpha}$ and $B_{\alpha}$ be defined and $\alpha<\mu$. Then $\left|S_{\alpha}\right|+\left|B_{\alpha}\right|<\mu$ (using b) and c)). Let $B^{0} \supseteq$ $B_{\alpha} \cup\{\alpha\}$ be any subset of $\mu$ with $\left|B^{0} \backslash B_{\alpha}\right|=\aleph_{0}$. By (3), we find $S^{0} \supseteq S_{\alpha}$ such that $M\left(S^{0}\right) \supseteq G_{B^{0}}$ and $\left|S^{0} \backslash S_{\alpha}\right|<\aleph_{1}$. Assuming $B^{n}, S^{n}$ are defined for $n<\omega$, we can find $B^{n+1} \supseteq B^{n}$ with $\left|B^{n+1} \backslash B^{n}\right|<\aleph_{1}$ such that $G_{B^{n+1}} \supseteq M\left(S^{n}\right)$, and $S^{n+1} \supseteq S^{n}$ with $\left|S^{n+1} \backslash S^{n}\right|<\aleph_{1}$ such that $M\left(S^{n+1}\right) \supseteq G_{B^{n+1}}$. Put $S_{\alpha+1}=\bigcup_{n<\omega} S^{n}$ and $B_{\alpha+1}=\bigcup_{n<\omega} B^{n}$. This completes the isolated step. In limit steps, we simply take unions. Since $M\left(S_{\mu}\right)=M$, we have $S_{\mu}=\sigma$ by (2).

Now, for each $\alpha<\mu$, we have the countable sets $C_{\alpha}=B_{\alpha+1} \backslash B_{\alpha}$ and $T_{\alpha}=$ $S_{\alpha+1} \backslash S_{\alpha}$, and the canonical projection $\pi_{\alpha}: M\left(S_{\alpha+1}\right) \rightarrow G_{C_{\alpha}}$. Let $\tau$ be the ordinal type of $\left(T_{\alpha},<\right)$, and fix an order-preserving bijection $i: \tau \rightarrow T_{\alpha}$.

Since $S_{\alpha} \cup\left(S_{\alpha+1} \cap \beta\right)$ is 'closed' for any $\beta \leq \sigma$ by (1), the part (2) yields that the chain $\left(N_{\beta} \mid \beta \leq \tau\right)$ of modules defined as $N_{\beta}=M\left(S_{\alpha} \cup\left(S_{\alpha+1} \cap i(\beta)\right)\right)$, for $\beta<\tau$, and $N_{\tau}=M\left(S_{\alpha+1}\right)$ is strictly increasing. Notice that $N_{0}=M\left(S_{\alpha}\right)$.

If we put $\bar{N}_{\beta}=\pi_{\alpha}\left[N_{\beta}\right]$ for all $\beta \leq \tau$, it follows that the strictly increasing chain $\left(\bar{N}_{\beta} \mid \beta \leq \tau\right)$ is a filtration of the free module $G_{C_{\alpha}}$ of countable rank. Moreover, for each $\beta<\tau$, we have $\bar{N}_{\beta+1}=\bar{N}_{\beta}+\left\langle\pi_{\alpha}\left(a_{i(\beta)}\right)\right\rangle$.

Finally, we recursively define the homomorphism $\psi: M \rightarrow R$. Let $\alpha<\mu$ and assume that $\psi \uparrow G_{B_{\alpha}}$ is constructed with the property $\psi\left(a_{\gamma}\right) \neq z_{\gamma}$ for all $\gamma \in S_{\alpha}$. By the already proven part for $\mu=\aleph_{0}$, we can define $\psi \uparrow G_{C_{\alpha}}$ in such a way that $\psi\left(\pi_{\alpha}\left(a_{\gamma}\right)\right) \neq z_{\gamma}-\psi\left(a_{\gamma}-\pi_{\alpha}\left(a_{\gamma}\right)\right)$ for all $\gamma \in T_{\alpha}$; observe that the right-hand side of the inequality is already defined since $a_{\gamma}-\pi_{\alpha}\left(a_{\gamma}\right) \in G_{B_{\alpha}}$. We immediately get $\psi\left(a_{\gamma}\right) \neq z_{\gamma}$ for all $\gamma \in S_{\alpha+1}$.

For the negative part, we start with an uncountable cardinal $\kappa$ and a $\kappa$-free group $G$ with the trivial dual, i.e. with the property $G^{*}:=\operatorname{Hom}(G, \mathbb{Z})=0$; here, $\kappa$-free means that any $<\kappa$-generated subgroup of $G$ is free. We will discuss the existence 
of such groups, as well as the question whether $G$ can be taken with $|G|=\kappa$, later on. Firstly, we show how the existence of such $G$ implies that $\kappa \notin \mathcal{S}$.

Let us denote by $\lambda$ the cardinality of $G$ and express $G$ as a quotient $F / K$ where $F$ is a free group of rank $\lambda$. Notice that $\lambda \geq \kappa$. Let $\pi: F \rightarrow F / K$ denote the canonical projection and let $\left\{e_{\alpha} \mid \alpha<\lambda\right\}$ be a set of free generators of the group $F$. For each $A \subseteq \lambda$, let $F_{A}$ denote the subgroup of $F$ generated by $\left\{e_{\alpha} \mid \alpha \in A\right\}$. We can w.l.o.g. assume that

$$
\operatorname{Im}\left(\pi \uparrow F_{\beta}\right) \subsetneq \operatorname{Im}\left(\pi \uparrow F_{\beta+1}\right) \text { for each ordinal } \beta<\lambda .
$$

The group $K$ is also free of rank $\lambda$. If it had a smaller rank, $G$ would have possessed a free direct summand - a contradiction with $G^{*}=0$. Let $\left\{k_{\beta} \mid \beta<\lambda\right\}$ denote a set of (free) generators of the group $K$. Consider the uncountable set

$$
S=\left\{\sum_{\alpha \in J_{\beta}} a_{\alpha \beta} x_{\alpha}=0 \mid \beta<\lambda, J_{\beta} \in[\lambda]^{<\omega},\left(\forall \alpha \in J_{\beta}\right)\left(a_{\alpha \beta} \in \mathbb{Z}\right), \sum_{\alpha \in J_{\beta}} a_{\alpha \beta} e_{\alpha}=k_{\beta}\right\}
$$

of homogeneous $\mathbb{Z}$-linear equations with the set $\left\{x_{\alpha} \mid \alpha<\lambda\right\}$ of variables. We will show that this is the desired counterexample.

First of all, $S$ does not have even a weakly nontrivial solution in $\mathbb{Z}$. Indeed, any such solution would define a nonzero homomorphism $\psi$ from $F$ to $\mathbb{Z}$ which is zero on $K$. Hence $\psi$ would provide for a nonzero homomorphism from $G$ to $\mathbb{Z}$, a contradiction.

On the other hand, we can show

Proposition 2.4. Any system $T \subseteq S$ with cardinality $<\kappa$ has a nontrivial solution in $\mathbb{Z}$.

Proof. Let $A \in[\lambda]^{<\kappa}$ be an infinite set such that whenever $x_{\alpha}$ appears in an equation from $T$ then $\alpha \in A$. Put $M=\operatorname{Im}\left(\pi \uparrow F_{A}\right)$.

Since $G$ is $\kappa$-free, $M$ is a free group (of infinite rank). Let $\sigma$ denote the ordinal type of $A$ and fix an order-preserving bijection $i: \sigma \rightarrow A$. For each $\alpha \leq \sigma$, set $M_{\alpha}=\left\langle\pi\left(e_{i(\beta)}\right) \mid \beta<\alpha\right\rangle$. Then $\left(M_{\alpha} \mid \alpha \leq \sigma\right)$ is a filtration of $M$ such that $M_{\alpha+1}=M_{\alpha}+\left\langle\pi\left(e_{i(\alpha)}\right)\right\rangle$ where $\pi\left(e_{i(\alpha)}\right) \notin M_{\alpha}$ for all $\alpha<\sigma$ (using $(*)$ ).

Applying Lemma 2.3 with $R=\mathbb{Z}$ and $z_{\gamma}=0$ for all $\gamma<\sigma$, we obtain a homomorphism $\psi: M \rightarrow \mathbb{Z}$ such that $\psi\left(\pi\left(e_{\alpha}\right)\right) \neq 0$ for all $\alpha \in A$. The assignment $x_{\alpha} \mapsto \psi\left(\pi\left(e_{\alpha}\right)\right), \alpha \in A$, is the desired nontrivial solution of the system $T$ in $\mathbb{Z}$.

Corollary 2.5. Let $\kappa$ be an uncountable cardinal. If there exists a $\kappa$-free group $G$ with $G^{*}=0$, then $\kappa \notin \mathcal{S} \cup \mathcal{W S}$.

\section{The CASE OF $\mathcal{W S}$}

For the weaker notion of nontrivial solvability, we have the following general result.

Proposition 3.1. Let $\kappa$ be an uncountable cardinal. The following conditions are equivalent:

(1) There exists a regular cardinal $\lambda \leq \kappa$ which is $\mathcal{L}_{\omega_{1} \omega}$-compact.

(2) There is a regular cardinal $\lambda \leq \kappa$ such that each group $A \in \operatorname{Ker} \operatorname{Hom}(-, \mathbb{Z})$ is the sum of its subgroups of cardinality $<\lambda$ which are contained in $\operatorname{Ker} \operatorname{Hom}(-, \mathbb{Z})$.

(3) For any nonempty system $S$ of homogeneous $\mathbb{Z}$-linear equations such that $S$ has no weakly nontrivial solution in $\mathbb{Z}$, and any $C \in[S]^{<\kappa}$, there exists $T \in[S]^{<\kappa}$ such that $C \subseteq T$ and $T$ has no weakly nontrivial solution in $\mathbb{Z}$. 
Proof. The equivalence of (1) and (2) follows directly from [1, Corollary 5.4]. Let us show that (2) is equivalent to (3). To this end, we are going to use the following two-way translation.

Given any system $S=\left\{k_{j}=0 \mid j \in J\right\}$ of homogeneous $\mathbb{Z}$-linear equations with the set $X$ of variables, we can build a group $A=F / K$ where $F$ is freely generated by the elements of the set $X$ and $K$ is generated by the set $\left\{k_{j} \mid j \in J\right\}$. Then $\operatorname{Hom}(A, \mathbb{Z})=0$ if and only if $S$ has no weakly nontrivial solution in $\mathbb{Z}$. On the other hand, for a given group $A$ and its presentation $F / K$ where $F$ is freely generated by a set $X$, the same equivalence holds for the system $S=\left\{k_{j}=0 \mid j \in J\right\}$ of homogeneous $\mathbb{Z}$-linear equations where $\left\{k_{j} \mid j \in J\right\}$ is a fixed set of generators of $K$ expressed as $\mathbb{Z}$-linear combinations of elements from the set $X$.

Proving $(2) \Longrightarrow(3)$, we start with a system $S$ and a set $C \in[J]^{<\kappa}$. Consider the group $A$ constructed for $S$ as in the previous paragraph, and let $Y_{0}$ denote the set of all the elements from $X$ appearing in equations $k_{j}=0, j \in C$.

Let $\mu \geq \lambda$ be a regular uncountable cardinal such that $|C|<\mu \leq \kappa$. Since $\operatorname{Ker} \operatorname{Hom}(-, \mathbb{Z})$ is closed under direct sums and quotients, and $\mu$ is regular, there exists, by $(2), G_{0} \in \operatorname{Ker} \operatorname{Hom}(-, \mathbb{Z})$ such that $G_{0}$ is a subgroup of $A,\left|G_{0}\right|<\mu$ and $Y_{0}+K:=\left\{y+K \mid y \in Y_{0}\right\} \subseteq G_{0}$. Now, take any $Y_{1} \in[X]^{<\mu}, Y_{0} \subseteq Y_{1}$ such that:

(a) $G_{0}$ is contained in the subgroup of $A$ generated by $Y_{1}+K$.

(b) There exists $C_{0} \in[J]^{<\mu}$ such that $\left\langle Y_{0}\right\rangle \cap K$ is contained in the subgroup of $K$ generated by $\left\{k_{j} \mid j \in C_{0}\right\}$, and $Y_{1}$ contains all the elements from $X$ appearing in equations $k_{j}=0, j \in C_{0}$.

For this $Y_{1}$, we obtain, using (2), a subgroup $G_{1}$ of $A$ with $\left|G_{1}\right|<\mu$, and so on.

After $\omega$ steps, we have the group $G=\sum_{n<\omega} G_{n} \in \operatorname{Ker} \operatorname{Hom}(-, \mathbb{Z})$ generated by $Y+K$ where $Y=\bigcup_{n<\omega} Y_{n} \in[X]^{<\mu}$. By the construction, we have also $G=\langle y+K|$ $y \in Y\rangle \cong\langle Y\rangle /\left\langle k_{j} \mid j \in \bigcup_{n<\omega} C_{n}\right\rangle$. Finally, we put $T=\left\{k_{j}=0 \mid j \in \bigcup_{n<\omega} C_{n}\right\}$.

Now, let us prove the implication $\neg(1) \Longrightarrow \neg(3)$. First, assume that $\kappa$ is not $\mathcal{L}_{\omega_{1} \omega^{-}}$compact. Following [1, Theorem 5.3] and its proof, we start with $A=\mathbb{Z}^{I} / \mathcal{F}$ where $\mathcal{F}$ is a $\kappa$-complete filter on $I$ which cannot be extended to an $\omega_{1}$-complete ultrafilter. From the latter part, it follows that $\operatorname{Hom}(A, \mathbb{Z})=0$. The $\kappa$-completeness of $\mathcal{F}$, on the other hand, assures that any subgroup of $A$ of cardinality $<\kappa$ can be embedded into $\mathbb{Z}^{I}$.

Consider a system $S$ of homogeneous $\mathbb{Z}$-linear equations associated to the group $A$ presented as $F / K$ where $F$ is freely generated by a set $X$. We can w.l.o.g. assume that no $x \in X$ is contained in $K$. Let $C \in[J]^{<\kappa}$ be non-empty. We shall show that the system $\left\{k_{j}=0 \mid j \in C\right\}$ has weakly nontrivial solution in $\mathbb{Z}$.

As in the proof of the other implication, we can possibly enlarge $C$ to some $D \subseteq J$ such that $|D| \leq|C|+\aleph_{0}$ and-denoting by $Y$ the set of all the elements from $X$ appearing in equations $k_{j}=0, j \in D-\langle y+K \mid y \in Y\rangle \cong\langle Y\rangle /\left\langle k_{j} \mid j \in D\right\rangle$. Let us denote the latter group by $H$ and fix an embedding $i: H \rightarrow \mathbb{Z}^{I}$ (which exists since $|H|<\kappa)$.

Let $y \in Y$ be any element appearing in (one of the) equations $k_{j}=0, j \in C$. Since $i(y+K) \neq 0$ there is a projection $\pi: \mathbb{Z}^{I} \rightarrow \mathbb{Z}$ such that $\pi i(y+K) \neq 0$. The assignment $x \mapsto \pi i(x+K)$ defines the desired weakly nontrivial solution of the system $\left\{k_{j}=0 \mid j \in C\right\}$ in $\mathbb{Z}$.

It remains to tackle the possibility that $\kappa$ is the least $\mathcal{L}_{\omega_{1} \omega}$-compact cardinal and $\kappa$ is singular. We know by [2] that $\gamma=c f(\kappa)$ is greater than or equal to the first measurable cardinal in this case. Let $\left(\kappa_{\alpha} \mid \alpha<\gamma\right)$ be an increasing sequence of cardinals $<\kappa$ converging to $\kappa$. 
Consider the group $A=\bigoplus_{\alpha<\gamma} A_{\alpha}$ where, for each $\alpha<\gamma, A_{\alpha} \in \operatorname{Ker} \operatorname{Hom}(-, \mathbb{Z})$ is not a sum of its subgroups of cardinality $<\kappa_{\alpha}$ which belong to $\operatorname{Ker} \operatorname{Hom}(-, \mathbb{Z})$. Assume, for the sake of contradiction, that (3) holds for the system $S$ of homogeneous $\mathbb{Z}$-linear equations associated to the group $A$ (more precisely, to its presentation $F / K)$.

By the definition of $A$, there exists, for each $\alpha<\gamma$, an element $a_{\alpha} \in A$ such that $a_{\alpha}$ is not contained in any subgroup $H$ of $A$ of cardinality $<\kappa_{\alpha}$ with the property $\operatorname{Hom}(H, \mathbb{Z})=0$.

We know that there is $C_{0} \in[J]^{<\kappa}$ and $Y_{0} \subseteq X$ consisting of the elements from $X$ appearing in the equations $k_{j}=0, j \in C_{0}$ such that $\left\{a_{\alpha} \mid \alpha<\gamma\right\} \subseteq\langle y+K| y \in$ $\left.Y_{0}\right\rangle \cong\left\langle Y_{0}\right\rangle /\left\langle k_{j} \mid j \in C_{0}\right\rangle$.

For this $C_{0}$, we obtain a corresponding $T_{0} \in[J]^{<\kappa}$ using (3). We continue by finding $C_{1} \in[J]^{<\kappa}$ and $Y_{1} \in[X]^{<\kappa}$ such that $T_{0} \subseteq C_{1}, Y_{0} \subseteq Y_{1}$ and $\langle y+K| y \in$ $\left.Y_{1}\right\rangle \cong\left\langle Y_{1}\right\rangle /\left\langle k_{j} \mid j \in C_{1}\right\rangle$, and so forth.

Put $T=\bigcup_{n<\omega} T_{n}=\bigcup_{n<\omega} C_{n}$ and $Y=\bigcup_{n<\omega} Y_{n}$. The system $\left\{k_{j}=0 \mid j \in T\right\}$ has cardinality $<\kappa$ (since $\gamma$ is uncountable) and it has no weakly nontrivial solution in $\mathbb{Z}$. Whence the subgroup $H=\langle y+K \mid y \in Y\rangle \cong\langle Y\rangle /\left\langle k_{j} \mid j \in T\right\rangle$ of $A$ belongs to $\operatorname{Ker} \operatorname{Hom}(-, \mathbb{Z})$. However, this is impossible since $a_{\alpha} \in H$ for $\alpha<\gamma$ satisfying $|H|<\kappa_{\alpha}$

In the proof above, we have actually showed a little bit more. In fact, we have the following

Theorem 3.2. Let $\kappa$ be a cardinal, and assume that $\kappa$ is not at the same time singular and the least $\mathcal{L}_{\omega_{1} \omega}$-compact cardinal. The following conditions are equivalent:

(1) $\kappa$ is $\mathcal{L}_{\omega_{1} \omega}$-compact.

(2) Any nonempty system $S$ of homogeneous $\mathbb{Z}$-linear equations has a weakly nontrivial solution in $\mathbb{Z}$ provided that each its nonempty subsystem of cardinality $<\kappa$ has one. In other words, $\kappa \in \mathcal{W S}$.

Proof. The implication ' $(1) \Longrightarrow(2)$ ' follows immediately from '(1) $\Longrightarrow(3)$ ' in Proposition 3.1 The other implication then from the first part of the proof of ' $\neg(1) \Longrightarrow \neg(3)$ ' in Proposition 3.1

\section{Concluding Remarks}

The problem of existence of $\kappa$-free groups with trivial dual turns out to be rather delicate. Under the assumption $\mathrm{V}=\mathrm{L}$ (even a much weaker one), there are $\kappa$-free groups with trivial dual for any uncountable cardinal $\kappa$. Moreover, if $\kappa$ is regular and not weakly compact, then the groups can be constructed of cardinality $\kappa$, see [3]. If $\kappa$ is singular or weakly compact, then $\kappa$-free implies $\kappa^{+}$-free. For more information on the topic, we refer to [4, Chapter VII]. Anyway, we have $\mathcal{S}=\mathcal{W} \mathcal{S}=\varnothing$ under $\mathrm{V}=\mathrm{L}$ by Corollary 2.5 .

In [5, Göbel and Shelah show in ZFC that $\aleph_{n}$-free groups with cardinality $\beth_{n}$ and trivial dual exist for all $0<n<\omega$. This is further generalized in [8], where Shelah proves in ZFC the existence of $\kappa$-free groups with trivial dual for any uncountable $\kappa<\aleph_{\omega_{1} \cdot \omega}$. On the other hand, he also shows (modulo the existence of a supercompact cardinal) that it is relatively consistent with ZFC that there is no $\aleph_{\omega_{1} \cdot \omega}$-free group with trivial dual.

By Corollary 2.5, we thus know in ZFC that $\kappa \notin \mathcal{S}$ for $\kappa<\aleph_{\omega_{1} \cdot \omega}$. However, we do not know what happens for larger cardinals $\kappa$ since the existence of a $\kappa$-free group with trivial dual is just a sufficient condition for $\kappa \notin \mathcal{S}$. We have only the upper

\footnotetext{
${ }^{1}$ Very heavy in content. Unpublished outside arXiv.org so far.
} 
bound given by Corollary 2.2. It might still be possible that $\mathcal{S}=\mathcal{W S}$ where for the latter class, we have a decent description in Theorem [3.2 As shown in [1, relative to the existence of a supercompact cardinal, there are models of ZFC where the smallest $\mathcal{L}_{\omega_{1} \omega}$-compact cardinal $\kappa$ is singular. In this only case, we cannot resolve the question whether $\kappa \in \mathcal{W S}$ although we conjecture that this is not the case, which would readily imply that at least $\mathcal{W S} \subseteq \mathcal{S}$ always holds.

A possible direction for further research is to investigate further what more can be proved in $\mathrm{ZFC}$ about the class $\mathcal{S}$.

Acknowledgement: I would like to thank Petr Glivický for a fruitful discussion about compactness problems in set theory.

\section{REFERENCES}

[1] J. Bagaria, M. Magidor, Group radicals and strongly compact cardinals, Trans. Amer. Math. Soc. 366 (2014), no. 1, 1857-1877.

[2] J. Bagaria, M. Magidor, On $\omega_{1}$-strongly compact cardinals, J. Symb. Log. 79 (2014), no. 1, $266-278$.

[3] M. Dugas, R. Göbel, Every cotorsion-free ring is an endomorphism ring, Proc. London Math. Soc. 45 (1982), 319-336.

[4] P. C. Eklof, A. H. Mekler, Almost Free Modules, Revised ed., North-Holland, New York 2002.

[5] R. Göbel, S. Shelah, $\aleph_{n}$-free modules with trivial dual, Res. Math. 54 (2009), 53-64.

[6] R. Göbel, J. Trlifaj, Approximations and Endomorphism Algebras of Modules, de Gruyter Expositions in Mathematics 41, 2nd revised and extended edition, Berlin-Boston 2012.

[7] H. Herrlich, E. Tachtsis, On the Solvability of Systems of Linear Equations over the Ring $\mathbb{Z}$ of Integers, Comment. Math. Univ. Carolin. 58 (2017), no. 2, 241-260.

[8] S. Shelah, Quite free complicated abelian group, pcf and black boxes, Sh:1028, arxiv.org/abs/1404.2775.

Charles University, Faculty of Mathematics and Physics, Department of Algebra, Sokolovská 83, 18675 Praha 8, Czech Republic

E-mail address: saroch@karlin.mff.cuni.cz 